\title{
CIRURGIA BARIÁTRICA E APNÉIA DO SONO
}

\author{
BARIATRIC SURGERY AND SLEEP APNEA
}

Reginaldo Ceneviva', Geruza A Silva², Melina M Viegas ${ }^{3}$, Ajith Kumar Sankarankutty ${ }^{1}$, Fernando Bahdur Chueire ${ }^{4}$

\begin{abstract}
${ }^{1}$ Docente. Disciplina de Gastroenterologia. Departamento de Cirurgia e Anatomia. ${ }^{2}$ Docente. Disciplina de Pneumologia. Departamento de Clínica Médica. ${ }^{3}$ Aluna especial. Curso de Pós-Graduação. Área de Clínica Cirúrgica. ${ }^{4}$ Médico Assistente. Disciplina de Nutrologia. Departamento de Clínica Médica. Faculdade de Medicina de Ribeirão Preto - USP.

CORRESPONDÊNICA: Reginaldo Ceneviva

Departamento de Cirurgia. Hospital das Clínicas da Faculdade de Medicina de Ribeirão Preto

Avenida dos Bandeirantes, 3900. CEP 14048-900. Monte Alegre, Campus de Ribeirão Preto-SP

e-mail: rceneviv@fmrp.usp.br
\end{abstract}

Ceneviva R, Silva GA, Viegas MM, Sankarankutty AK, Chueire FB. Cirurgia bariátrica e apnéia do sono. Medicina (Ribeirão Preto) 2006; 39 (2): 235-245.

RESUMO: A obesidade constitui um problema médico-social importante por sua prevalência alta e crescente e sua gravidade. Tem características epidêmicas e pode favorecer ou agravar hipertensão arterial, diabetes mellitus, artropatias degenerativas, apnéia do sono, e disfunções respiratórias e cardiovasculares. A piora da qualidade de vida, a redução da expectativa de vida e a alta taxa de fracasso dos tratamentos conservadores são fatores que reforçam a indicação de tratamento cirúrgico da obesidade mórbida. A indicação cirúrgica baseia-se na análise conjunta de múltiplos aspectos clínicos, incluindo a falha do tratamento conservador e a avaliação psicológica. São parâmetros importantes o índice de massa corpórea acima de $40 \mathrm{~kg} / \mathrm{m}^{2}$, ou acima de $35 \mathrm{~kg} / \mathrm{m}^{2}$ na presença de doença agravada ou causada pela obesidade. As modalidades cirúrgicas, de acordo com seu objetivo fundamental, são: restritivas, disabsortivas e mistas. Como para outras co-morbidades, é importante o preparo pré-e pós operatório adequado dos obesos com apnéia obstrutiva do sono (AOS) grave. Além de cuidados especiais relativos à ventilação é recomendado que o paciente perca de 10 a $15 \%$ de seu peso antes da intervenção cirúrgica. Redução de $10 \%$ do peso original corresponde geralmente à diminuição de cerca de $25 \%$ no índice de apnéia e hipopnéia. Os efeitos sobre a AOS são tanto melhores quanto maiores forem a redução do peso e a manutenção dessa perda. Resultados de meta-análise demonstram que a perda efetiva de peso após cirurgia bariátrica resolve as co-morbidades na maioria dos pacientes, com cura ou melhora da AOS em $86,3 \%$ dos pacientes. No Brasil dá-se preferência à gastroplastia vertical com bandagem e derivação gástrica em Y de Roux (técnica de Fobi-Capella) por conseguir perda de $40 \%$ do peso inicial, mantida a longo prazo e por não produzir alterações nutricionais e metabólicas importantes.

Descritores: Obesidade; cirurgia. Cirurgia Bariátrica. Síndromes da Apnéia do Sono.

\section{1- INTRODUÇÃO}

A obesidade é uma doença que se caracteriza por acúmulo de gordura corporal, com prejuízo da saúde. A obesidade e seus diferentes graus podem ser definidos com base no índice de massa corporal (IMC) que corresponde à razão do peso corporal (em kg) pelo produto da altura pela altura (em metros). Define obesidade IMC igual ou maior que $30 \mathrm{~kg} / \mathrm{m}^{2}$.

A obesidade constitui um problema médico-social importante por sua gravidade e prevalência alta e crescente; por suas proporções epidêmicas é também 
um problema de saúde pública quase universal, sobretudo nos países ocidentais. No Brasil, de acordo com dados do IBGE (2004), a obesidade atinge $11 \%$ da população, correspondendo a 10,5 milhões de pessoas ${ }^{1}$.

Quando o excesso de peso ultrapassa valores altos, com IMC $\geq 40 \mathrm{~kg} / \mathrm{m}^{2}$, a obesidade passa a ser considerada uma doença grave, em função da alta freqüência de associação com doenças que são causadas ou agravadas por ela; corresponde à obesidade grau III, também chamada obesidade mórbida. Entre as co-morbidades mais freqüentes encontram-se hipertensão arterial, diabetes mellitus tipo II, apnéia obstrutiva do sono, artropatias degenerativas, dislipidemia, coronariopatia, disfunções respiratórias, colelitíase e desajustes psicossociais.

O excesso de peso corporal e as co-morbidades resultam na queda da qualidade de vida e na redução da expectativa de duração da vida. Peeters et al. encontraram em obesos mórbidos da população branca redução de 7 anos na duração da vida dos homens e de 5 anos das mulheres ${ }^{2}$.

O tratamento da obesidade objetiva a melhora da saúde e da qualidade de vida, mediante redução do peso corporal, suficiente para curar ou melhorar as co-morbidades e promover o bem estar psicológico ${ }^{3}$. Isso constitui um atributo da cirurgia bariátrica, visto que a obesidade mórbida permanece largamente refratária à terapêutica dietética e medicamentosa ${ }^{3 / 7}$.

Como orientação geral as indicações para o tratamento cirúrgico da obesidade baseiam-se sobretudo na gravidade associada ao IMC ou ao excesso de peso e à presença de co-morbidades:

1) $\mathrm{IMC} \geq 40 \mathrm{~kg} / \mathrm{m}^{2}$ ou excesso de peso $\geq 45 \mathrm{~kg}$

2) IMC $\geq 35 \mathrm{~kg} / \mathrm{m}^{2}$ ou excesso de peso $\geq 40 \mathrm{~kg}$ com co-morbidades resultantes ou agravadas pela obesidade.

Essas indicações não são absolutas nem devem ser tomadas isoladamente, mas complementadas com avaliação individualizada por equipe multidisciplinar que envolve o concurso de clínicos, cirurgiões, nutricionistas, psicólogos e, eventualmente, de cardiologistas, psiquiatras e fisioterapeutas.

Os requisitos necessários para a indicação cirúrgica a partir da avaliação clínica e laboratorial individualizada são:

1) Ausência de endocrinopatias cujo tratamento pode, por si, favorecer o emagrecimento adequado
2) Falha inconteste de terapêutica não-cirúrgica bem orientada por período mínimo de dois anos

3) Risco cirúrgico, pelo menos, satisfatório

4) Capacidade de entender a cirurgia e suas conseqüências

5) Motivação e estrutura psíquica favorável.

O paciente deve estar ciente dos possíveis riscos do tratamento cirúrgico e disposto a colaborar no período pós-operatório com os esquemas de dieta fracionada e fisioterapia, além do controle clínico e laboratorial periódico após a alta hospitalar.

São contra-indicações: pneumopatias e cardiopatias graves, cirrose hepática, distúrbios psiquiátricos, insuficiência renal e dependência de álcool ou de drogas. A prevalência das co-morbidades tem aumentado à medida que a obesidade mórbida continua a aumentar. A apnéia obstrutiva do sono é uma das mais freqüentes.

\section{2- APNÉIA OBSTRUTIVA DO SONO E OBE- SIDADE}

O fator de risco mais importante para a apnéia obstrutiva do sono (AOS) é a obesidade, especialmente com acúmulo de gordura na porção alta do abdome. Pelo menos 60 a $70 \%$ dos pacientes com AOS são obesos $^{8}$. A incidência da AOS entre pacientes com obesidade grau III é 12 a 30 vezes maior que na população geral ${ }^{9}$. Investigações clínicas e laboratoriais mostram que a incidência da AOS é de 42 a $48 \%$ nos homens obesos ${ }^{10}$, e 8 a $38 \%$ nas mulheres obesas ${ }^{11,12}$. A Figura 1 ilustra a ocorrência de co-morbidades em 92 pacientes consecutivos com obesidade mórbida submetidos à cirurgia bariátrica no Hospital das Clínicas da Faculdade de Medicina de Ribeirão Preto da Universidade de São Paulo, com apnéia do sono em 64,5\% dos pacientes*.

A fisiopatologia da AOS é complexa e incompletamente compreendida. No estreitamento das vias aéreas superiores (VAS) podem estar envolvidos fatores não específicos como deposição de gordura no pescoço ou morfologia anormal da via aérea superior. As alterações anatômicas e funcionais da faringe, o sistema nervoso central, a gordura central (nas vísceras e no tronco) e a leptina (hormônio produzido pela gordura visceral) provavelmente interagem no desenvolvimento da AOS em pessoas obesas. Por outro lado

\footnotetext{
* Dados apresentados no IX Congresso Brasileiro de Nutrologia, São Paulo. 8 a 10 de setembro de 2005. Ceneviva R, Santos JE, Pitanga KC, Souza JAG, Mota GA, Chueire FB. Resultados da gastroplastia vertical e derivação gastrojejunal em Y de Roux em 92 pacientes consecutivos.
} 


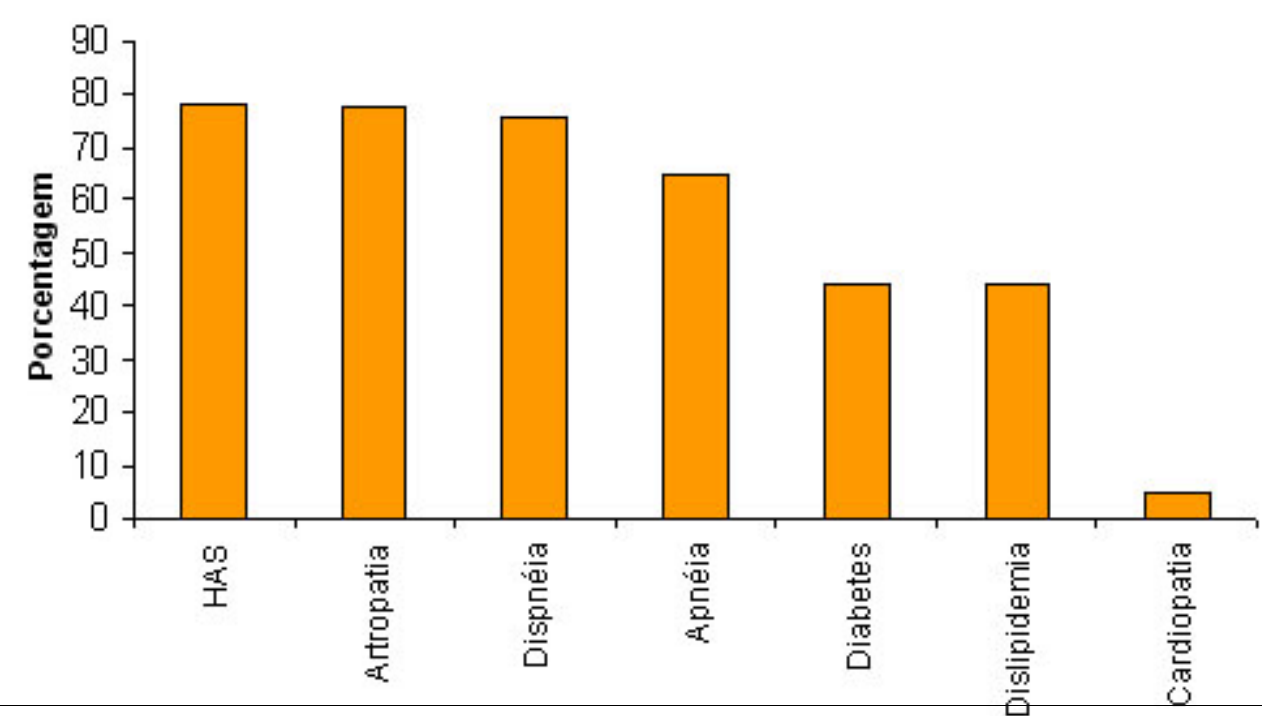

Figura 1: Co-morbidades em 92 pacientes com obesidade mórbida.

a AOS pode predispor indivíduos ao agravamento da obesidade em conseqüência da deprivação do sono, sonolência diurna e prejuízo no metabolismo ${ }^{5}$.

A redução na área de secção transversal da faringe, resultante do depósito de gordura, é considerada o mecanismo inicial predominante levando à obstrução das VAS no obeso ${ }^{13,14}$. O comprometimento funcional dos músculos dilatadores das VAS é particularmente importante. Alterações nos mecanismos de controle respiratório têm sido também encontradas em associação com a AOS, incluindo piora no comando químico e nas respostas de carga inspiratória e anormalidade dos reflexos protetores das VAS ${ }^{15}$. Essas alterações levam à redução da resposta à hipercapnia e à elevação da pressão parcial de $\mathrm{CO}_{2}$ arterial.

A estimativa da gordura corporal mediante impedância bioelétrica foi demonstrada ser um fator preditivo da $\operatorname{AOS}^{16}$. A circunferência do pescoço mostrou ser um preditor mais útil do que o grau de obesidade ${ }^{17,18}$.

\section{3- CIRURGIA BARIÁTRICA NO TRATAMEN- TO DA APNÉIA OBSTRUTIVA DO SONO}

\section{1- Avaliação pré-operatória}

A condução adequada dos pacientes obesos com apnéia obstrutiva do sono requer uma avaliação préoperatória rigorosa dos riscos anestésicos e operatórios. O período pós-operatório imediato constitui um desafio adicional.
Em virtude da alta prevalência de AOS em pacientes obesos, o uso rotineiro da polissonografia antes da cirurgia bariátrica tem sido recomendado com o objetivo de identificar todos os portadores da síndrome ${ }^{19}$.

A avaliação das vias aéreas superiores e da função pulmonar é indispensável para orientar a prevenção de complicações durante a anestesia e no período pós-operatório imediato. Os pacientes obesos com AOS têm o risco de sofrer descompensações respiratórias com agravamento da hipoventilação e de distúrbios respiratórios restritivos. O aumento da massa corpórea resulta no aumento do consumo de oxigênio e da produção de dióxido de carbono, pelo que complicações respiratórias agudas pós-operatórias são duas vezes mais freqüentes nos obesos do que nos nãoobesos $^{20}$. Em obesos sem doença pulmonar não é usual encontrar-se alteração na função pulmonar. A alteração respiratória típica do obeso mórbido, com ou sem AOS, é restritiva, podendo associar-se à hipoxemia crônica $\left(\mathrm{paO}_{2} \leq 65 \mathrm{mmHg}\right)$ e à hipoventilação $\left(\mathrm{paCO}_{2} \geq 45 \mathrm{mmHg}\right)$. A oximetria de pulso é um método de triagem razoável para essa alteração respiratória.

Em alguns pacientes a gravidade da AOS contra-indica a cirurgia e nessa situação deve-se tentar preparar adequadamente o paciente mediante a utilização de ventilação não-invasiva com Bi-PAP ou CPAP e emagrecimento suficiente para melhora substancial do índice de apnéias. 
A obesidade tem sido associada à dificuldade para laringoscopia, para intubação traqueal e para ventilação com máscaras faciais; essa dificuldade deve ser identificada no período pré-operatório para a tomada oportuna das medidas mais adequadas de prevenção.

A avaliação pré-operatória das vias aéreas deve ser complementada pelo exame do pescoço; a circunferência do pescoço de aproximadamente $40 \mathrm{~cm}$ já implica na probabilidade de intubação traqueal difícil. Esse risco é de $35 \%$ se o pescoço tiver circunferência de $60 \mathrm{~cm}^{20}$. Embora a abordagem das vias aéreas possa ser um desafio nessa população, uma boa avaliação pré-operatória permite minimizar os riscos potenciais. Problemas cardiovasculares e pulmonares também podem ser identificados e tomados em consideração no planejamento da anestesia e da cirurgia. Um dos fatores que colaboram para a boa qualidade dos cuidados ao paciente com AOS é o conhecimento que o próprio paciente tem de sua doença antes de se iniciar a anestesia. A postergação da cirurgia para permitir o diagnóstico formal em todos os pacientes com suspeita de AOS é aconselhável. A utilização de CPAP e/ou a perda de 10 a $15 \%$ do peso corpóreo antes da cirurgia minimizam significativamente o risco de complicações respiratórias ou cardiovasculares nessa população ${ }^{20 .}$

A perda de peso faz parte do tratamento de todos os pacientes obesos que têm apnéia do sono. Pacientes que perdem pouco peso com os programas clínicos, ou que são muito obesos mesmo após a perda, podem não apresentar melhora da apnéia ${ }^{21,22,23}$. Além disso, a taxa de sucesso dos programas clínicos de perda de peso de longo termo é desapontadora, o que favorece a opção pelo tratamento cirúrgico ${ }^{24}$.

\section{2- Implicações durante a recuperação anesté- sica e no pós-operatório imediato}

Terminada a cirurgia bariátrica os pacientes, sobretudo aqueles com AOS, requerem atenção especial no centro de recuperação anestésica, com monitoramento e avaliação periódica das VAS. A sonda traqueal, usada para ventilação durante a anestesia, somente deve ser retirada após plena reversão anestésica e com o paciente em boas condições de ventilação espontânea.

Dentre os admitidos no centro de recuperação anestésica aproximadamente um em 1500 pacientes necessitará re-intubação. Pacientes obesos e com AOS merecem maior atenção, também porque neles a re-intubação traqueal é mais difícil ${ }^{25}$.
A sedação farmacológica pode diminuir o tônus da musculatura faríngea superior. Embora esse tônus diminuído possa ser bem tolerado na maioria dos pacientes, a combinação da sedação e AOS pode comprometer a permeabilidade das vias aéreas superiores. Oxigênio contínuo por catéter nasal ou máscara facial para manter a saturação de oxigênio arterial adequada e uso de CPAP em pacientes que exibem colapso das vias aéreas superiores, podem ser necessários.

Os pacientes com AOS devem permanecer enquanto hospitalizados, sobretudo no pós-operatório imediato, com a cabeça elevada em pelo menos $30^{\circ}$ com o objetivo de melhorar a estabilidade das vias aéreas.

A destinação do paciente, após a completa recuperação anestésica, depende de vários fatores que incluem a avaliação da gravidade da AOS, mediante o índice pré-operatório de apnéia -hipopnéia e a dependência de CPAP para dormir. Outros fatores considerados são a presença de insuficiência cardíaca, doença pulmonar subjacente, grau de obesidade e o tipo de cirurgia. Pacientes com AOS leve, na ausência de co-morbidades ou com co-morbidades leves, e que se submeteram a procedimentos cirúrgicos de pequeno porte podem ter alta hospitalar no mesmo dia. Por outro lado, pacientes com AOS grave que requerem CPAP regularmente ou aqueles com co-morbidades graves ou diversas necessitam atendimento em unidade de cuidados intermediários ou em centro de cuidados intensivos, na dependência da natureza e do porte do procedimento cirúrgico. Os pacientes que se colocam em situação intermediária a esses dois grupos podem ser encaminhados para a enfermaria cirúrgica.

Outros problemas que freqüentemente acompanham a AOS e a obesidade, como hipertensão arterial e insuficiência cardíaca, também merecem monitoramento e os devidos cuidados após a cirurgia, principalmente no que diz respeito à hidratação pós-operatória ${ }^{26}$. Embora a diminuição da dor seja um dos objetivos da assistência médica, o uso de narcóticos é especialmente perigoso em pacientes com AOS. Após o uso de anestésicos gerais o paciente com AOS apresenta, nos primeiros dias, propensão ao aumento do sono REM (com movimentos rápidos dos olhos), o que favorece a ocorrência de paralisia do músculo genioglosso e queda da língua para o espaço faringeano, comprometendo a ventilação. Os despertares que interrompem essa sequiência de eventos ficam prejudicados pela administração de narcóticos ou de sedativos. A depressão respiratória, com possibilidade de 
indução de parada cardíaca, pode ser evitada pela elevação do tronco $\left(30^{\circ}\right)$, uso de CPAP, e analgésicos não- esteróides no tratamento da dor. Pacientes muito obesos podem apresentar queda brusca da saturação de oxigênio e a parada cardíaca pode ocorrer de maneira surpreendentemente rápida. Em situações graves a intubação traqueal, ou mesmo a traqueostomia, pode ser necessária.

A deambulação precoce e o posicionamento correto do paciente no leito são muito importantes na profilaxia de complicações pulmonares e de trombose venosa profunda, mas podem ser difíceis sobretudo em pacientes muito obesos e nos portadores de limitações impostas por artropatias dos membros inferiores. A fisioterapia é de importância fundamental na assistência aos pacientes antes e após a cirurgia bariátrica.

\section{4- TÉCNICAS CIRÚRGICAS}

As técnicas cirúrgicas utilizadas atualmente para o tratamento da obesidade mórbida são de três tipos: restritivas, disabsortivas e mistas.

As restritivas visam, mediante saciedade precoce favorecida por redução da capacidade gástrica, diminuir o volume de alimentos ingeridos; as disabsortivas, mediante exclusão de segmento do intestino delgado do trânsito, visam reduzir a absorção de alimentos, e as mistas associam restrição mecânica ao bolo alimentar e má absorção intestinal.

\section{1- Técnicas restritivas}

As técnicas restritivas mais utilizadas são o balão intragástrico, a banda gástrica ajustável laparoscópica e a gastroplastia vertical com bandagem (Figura 2).

A aplicação do balão intragástrico prescinde de intervenção cirúrgica .

Confeccionado com silicone, o balão é introduzido e insuflado no estômago por método endoscópico; com volume de 500 a $800 \mathrm{ml}$ de material aquoso, ocupa espaço no estômago proporcionando saciedade precoce. É um procedimento temporário, após a sua retirada o paciente volta à tendência de ganhar peso; tem sido utilizado para substituir o tratamento conservador, sobretudo como preparo para a cirurgia bariátrica. Permite ao paciente manter suas atividades sociais e laborativas.

A banda gástrica ajustável laparoscópica, proposta por Kuzmak ${ }^{27}$ e padronizada por Belachew ${ }^{28}$ e Favretti ${ }^{29}$, consiste em uma banda inflável de silicone aplicada por via laparoscópica na porção alta do estômago, estreitando-a, de maneira a criar uma câmara gástrica proximal pequena que se comunica com a porção distal restante do estômago através de orifício de passagem regulável pelo grau de insuflação da banda.

Esta modalidade tem os mesmos princípios da gastroplastia vertical à Mason ${ }^{30}$, permitindo, pela passagem lenta dos alimentos pelo orifício de comuni-
A

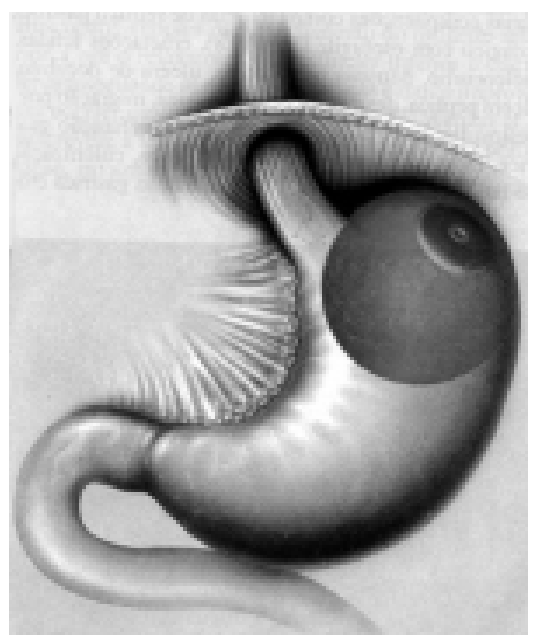

B

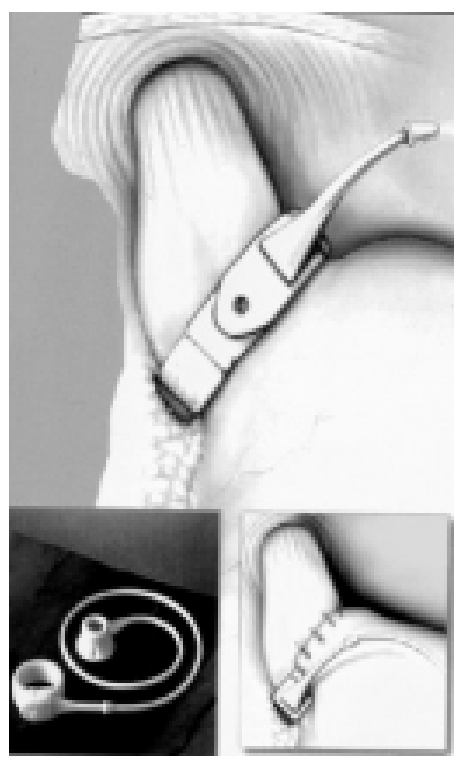

C

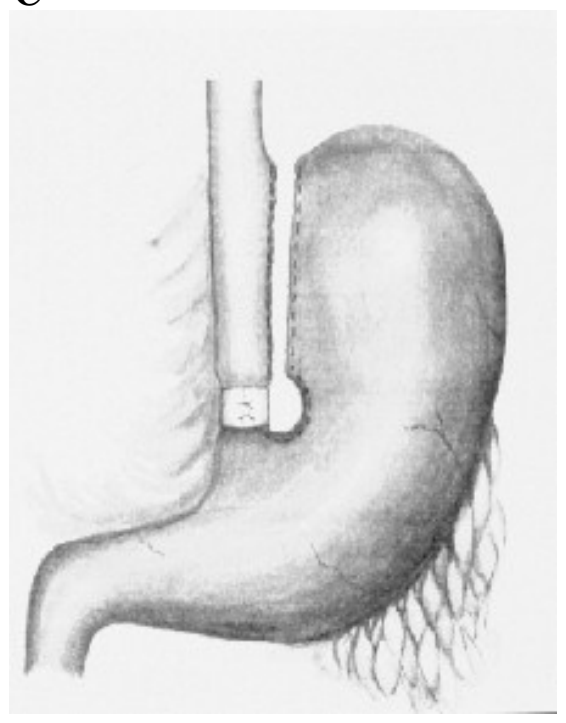

Figura 2: Técnicas restritivas: A - balão intragástrico, B - banda gástrica ajustável e C - gastroplastia vertical com bandagem 
cação, que a câmara gástrica proximal se distenda com quantidade pequena de alimentos resultando em saciedade precoce.

A banda gástrica ajustável induz, em média, perda de $25 \%$ a $30 \%$ do peso original; tem o mesmo inconveniente da gastroplastia vertical a Mason de os pacientes retomar ganho de peso à medida que passam a ingerir alimentos líquiidos hipercalóricos, com alta tolerância. Ingestão de 5000 a 6000 calorias por dia é freqüiente.

\section{2- Técnicas disabsortivas}

Os procedimentos cirúrgicos exclusivamente disabsortivos têm como principal representante a derivação jejuno-ileal, realizada sobretudo mediante a técnica de Payne na década de $1960{ }^{31}$. (Figura 3).

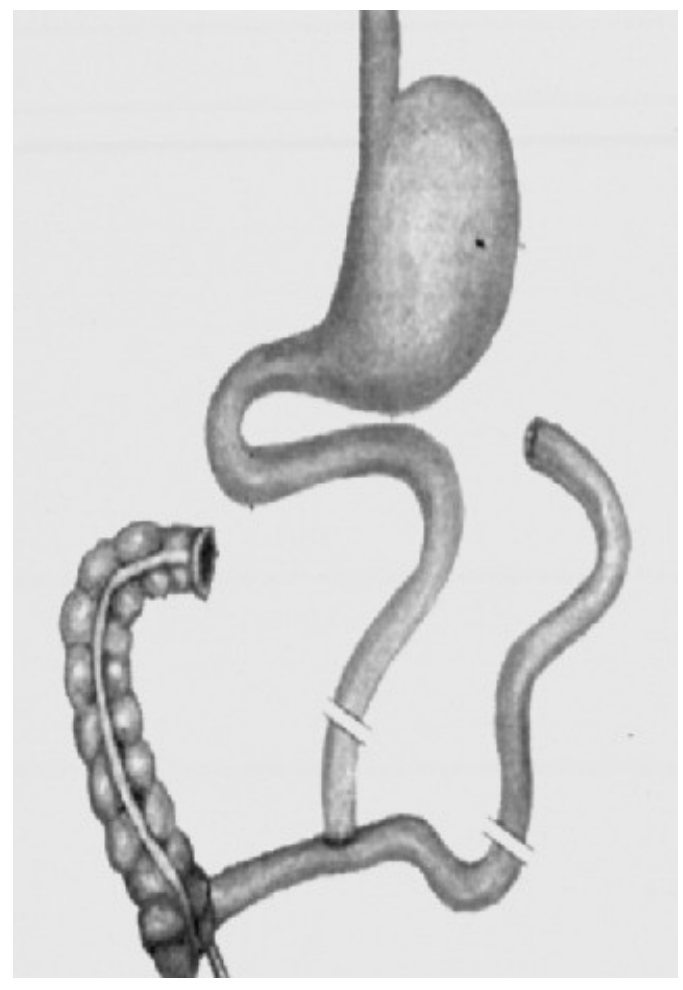

Figura 3: Técnica disabsortiva: derivação jejuno-ileal à Payne

Envolve a exclusão de quase todo o intestino delgado, permanecendo no trânsito alimentar apenas $35 \mathrm{~cm}$ de jejuno proximal e $10 \mathrm{~cm}$ do íleo distal. A derivação jejuno-ileal foi abandonada em decorrência das freqüentes e não raramente graves alterações nutricionais e metabólicas secundárias à má-absorção, sendo substituída pelas gastroplastias e derivações bíliopancreáticas.

\section{3- Técnicas mistas}

As técnicas mistas principais estão representadas na Figura 4.
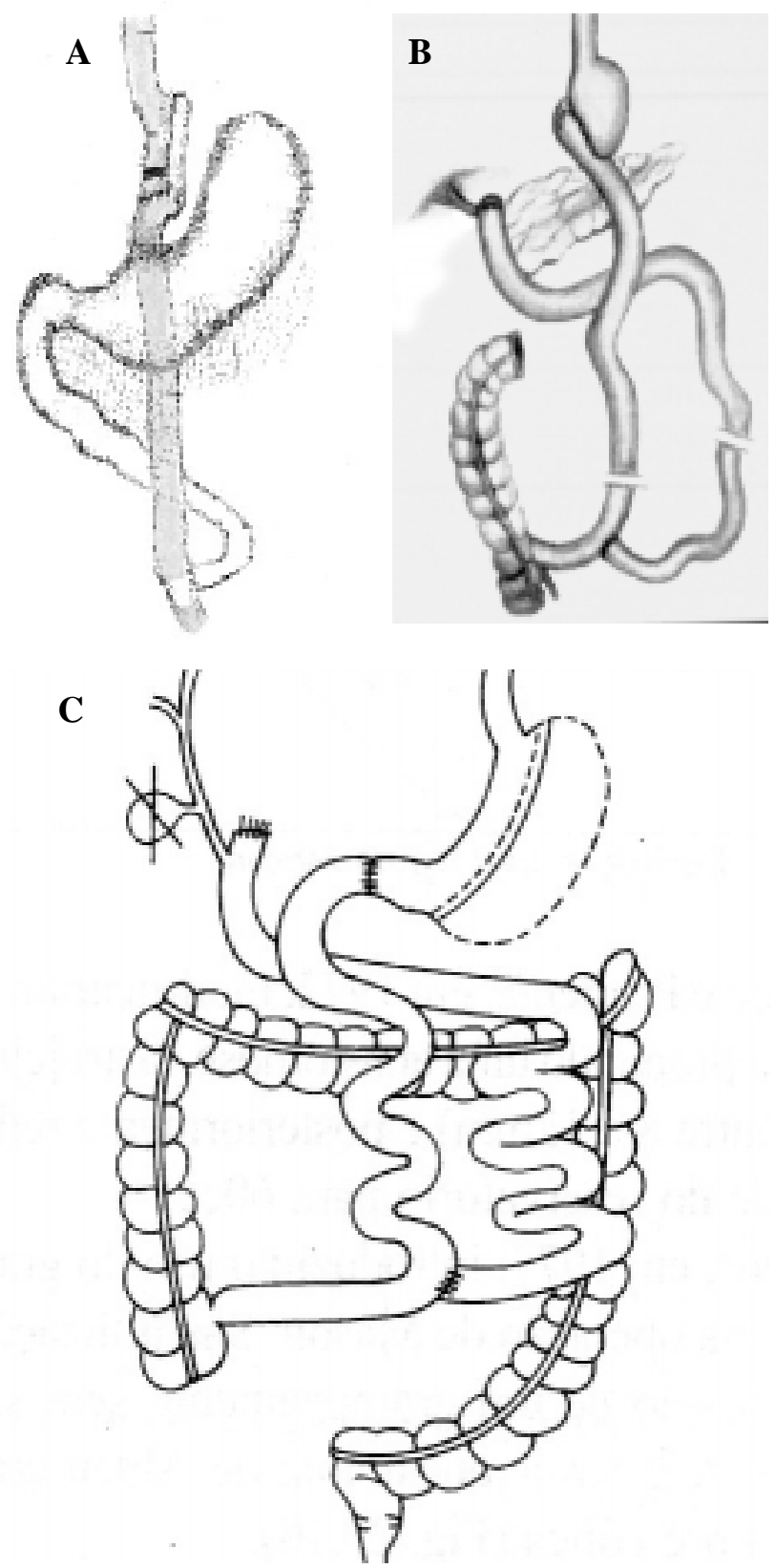

Figura 4: Técnicas mistas: A - Fobi-Capella, B - Scopinaro e C - Marceau-Hess

\subsection{1- Técnicas mistas predominantemente res- tritivas}

A operação de Fobi-Capella é a modalidade da gastroplastia vertical com derivação gástrica mais freqüientemente utilizada ${ }^{32}$. Essa operação envolve a separação de câmara gástrica proximal junto à cárdia, 
com capacidade de 20 a $30 \mathrm{ml}$, do restante do estômago e a anastomose com alça jejunal em Y de Roux de $100 \mathrm{~cm}$; assim a maior parte do estômago, o duodeno e $50 \mathrm{~cm}$ do jejuno proximal ficam excluídos do trânsito alimentar, representando o pequeno componente disabsortivo. O componente restritivo corresponde à redução do reservatório gástrico e à restrição ao seu esvaziamento pelo emprego de um anel de contenção de silicone com circunferência de $6,2 \mathrm{~cm}$, determinando uma saída de aproximadamente $1,5 \mathrm{~cm}$ de diâmetro.

Essa modalidade terapêutica tem-se demonstrado eficaz na indução da perda de peso e na manutenção de perda satisfatória, a longo prazo. A perda média do excesso de peso tem sido referida como de $50 \%$ a $70 \%$ após 14 anos; Ceneviva et al* e Garrido et al. ${ }^{33}$ relatam redução média de $40 \%$ do peso préoperatório, com estabilização a partir de um ano.

Wittgrove e Clark adaptaram a derivação gástrica à via laparoscópica ${ }^{34}$. Esse tipo de abordagem deve ser limitado a cirurgiões com grande experiência em cirurgia videolaparoscópica; na experiência inicial de Garrido et al. há sugestão de que a redução ponderal média aos 6 e aos 12 meses é discretamente menor do que com o método convencional ${ }^{33}$.

\subsection{2- Técnicas mistas predominantemente di- sabsortivas}

A derivação bílio-pancreática pela técnica de Scopinaro é uma variante mais moderna da técnica de Payne ${ }^{35}$. Esse procedimento é misto, com componente restritivo menos significante que o disabsortivo e envolve uma gastrectomia subtotal distal, mantendo coto gástrico com capacidade de 200 a 500 ml; o trânsito alimentar é reconstituído mediante anastomose do coto gástrico com os $2,5 \mathrm{~m}$ do íleo terminal. A alça exclusa do trânsito, que corresponde a pouco menos de $60 \%$ do intestino delgado, drena a secreção bíliopancreática para encontrar o bolo alimentar através de uma anastomose feita no íleo a $50 \mathrm{~cm}$ da válvula íleo-cecal. Com o passar do tempo e com a adaptação gástrica o paciente passa a comer à vontade e a cirurgia passa a ser exclusivamente disabsortiva.

Scopinaro relata perda de $72 \%$ do excesso de peso corporal, mantida por 18 anos; esse é o melhor resultado quanto à perda de peso e à manutenção da perda, apesar do paciente poder ingerir grande quantidade de alimentos ${ }^{36}$. À médio e à longo prazo podem-se desenvolver complicações como desnutrição protéica em $15 \%$ dos casos, osteoporose, osteomalácea e hipovitaminoses pelo que os pacientes exigem cuidados nutricionais permanentes, necessitando receber suple- mento de cálcio e vitaminas, sobretudo vitamina D.

Na técnica de Scopinaro modificada por Marceau e Biron (duodenal switch) a gastrectomia parcial é vertical, com manutenção do piloro; a alça intestinal que drena a secreção bílio-pancreática é anastomosada à alça ileal que recebe o bolo alimentar a $100 \mathrm{~cm}$ da válvula íleo-cecal em vez de $50 \mathrm{~cm}$, dobrando assim o comprimento do segmento ileal comum absortivo ${ }^{37}$. Essa operação mantém os bons resultados da técnica de Scopinaro quanto à redução de peso e reduz os índices de alterações nutricionais e metabólicas ${ }^{38}$.

\section{5- RESULTADOS DA CIRURGIA BARIÁTRICA}

A operação de Fobi-Capella (gastroplastia vertical com bandagem e derivação gástrica em Y de Roux), a bandagem gástrica ajustável laparoscópica e a operação de Scopinaro (derivação bílio-pancreática) são os procedimentos mais utilizados no tratamento intervencionista da obesidade mórbida. A possibilidade de ganho de peso em face da ingestão de alimentos líquiidos de alto teor calórico limita a indicação para pacientes que necessitam de grande perda ponderal e para os que não conseguem manter hábitos alimentares adequados.

A derivação bílio-pancreática pode ser uma boa opção para pacientes muito obesos e tem sido um dos procedimentos mais utilizados na Europa. A preferência no Brasil e nos USA é pela operação de FobiCapella em função de seus bons resultados quanto à perda de peso mantidos a longo prazo, sem os inconvenientes dos prejuízos nutricionais e metabólicos da derivação bílio-pancreática.

A morbidade do tratamento cirúrgico da obesidade do tipo III em geral acontece em 10 a $30 \%$ dos casos e envolve complicações próprias do tipo de cirurgia empregado e as complicações comuns às várias modalidades cirúrgicas, em princípio, favorecidas ou agravadas pela obesidade.

Problemas pulmonares, como atelectasia e pneumonia, não são raros (8\%) e geralmente não são graves. A embolia pulmonar é rara (1\%) e grave. A mortalidade operatória geral é de aproximadamente $1 \%$. A redução do peso corpóreo intensa e persistente acompanha-se geralmente da cura ou da melhora das doenças associadas à obesidade e está relacionada com altos índices de readaptação psicológica e social ${ }^{39}$. A Figura 5 ilustra resultados da cirurgia de Fobi-Capella realizada em 92 pacientes com obesidade mórbida no Hospital das Clínicas da Faculdade de Medicina de Ribeirão Preto da Universidade de São Paulo, com seguimento de 2 meses a 5 anos. 


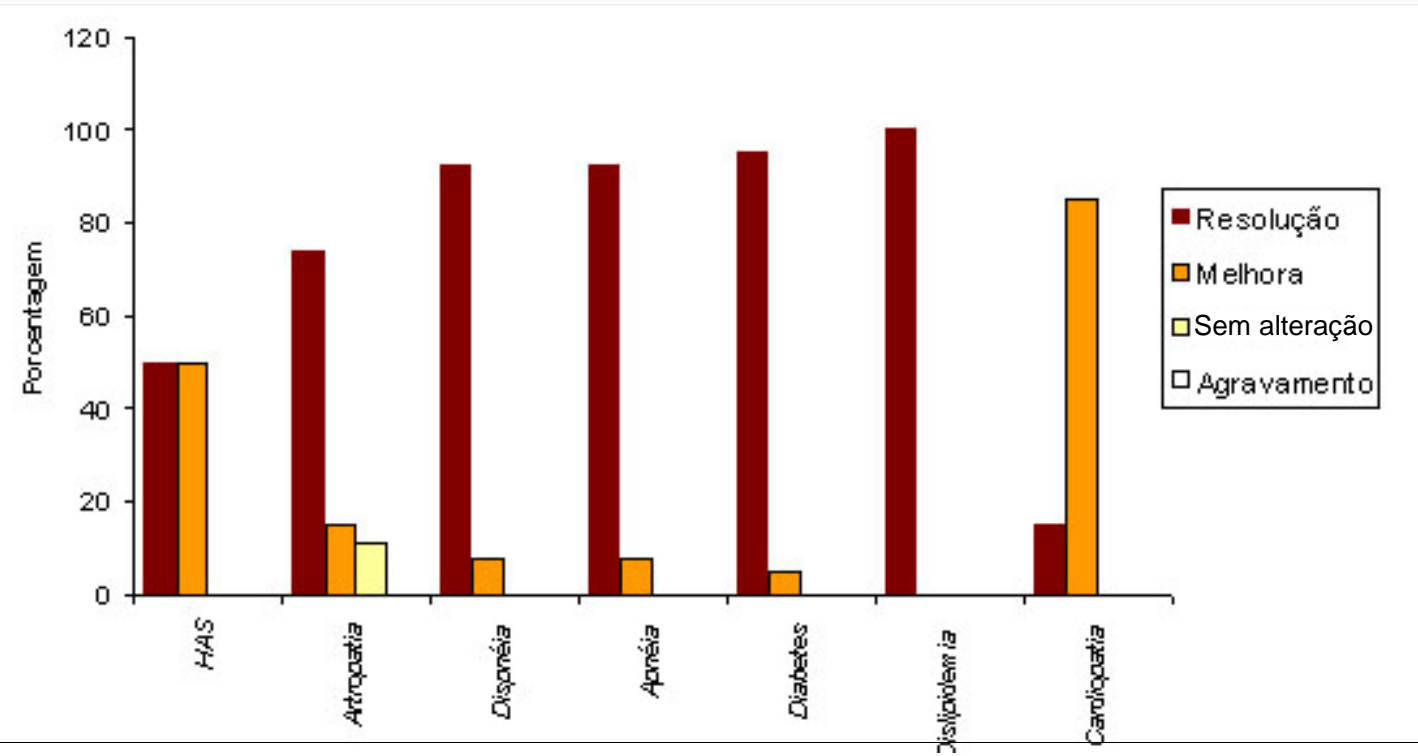

Figura 5: Evolução de doenças associadas à obesidade mórbida em 92 pacientes após cirurgia de Fobi-Capella

A evolução das doenças associadas à obesidade mórbida após a cirurgia bariátrica foi significantemente satisfatória. Houve desaparecimento dos sintomas da apnéia do sono em $92 \%$ e melhora em $8 \%$ dos pacientes operados*.

O prognóstico depende da seleção adequada do paciente e do tipo de cirurgia. A seleção do paciente envolve avaliação psicológica criteriosa visando definir se o paciente tem estrutura psíquica favorável para suportar as transformações radicais de comportamento impostas pela operação.

O tratamento cirúrgico e a perda de peso geralmente acarretam: diminuição da depreciação da imagem corporal, melhor disposição para o trabalho, melhora das atividades físicas, sociais e sexuais, e aumento da auto-estima.

\section{6- RESULTADOS DACIRURGIABARIÁTRICANO TRATAMENTO DAAPNÉIA OBSTRUTIVADO SONO}

As alterações moderadas no peso corporal podem resultar em significantes oscilações na prevalência de distúrbios respiratórios; Peppard et al., com base em estudo longitudinal realizado em 690 habitantes de Wisconsin, demonstraram que o aumento de $10 \%$ no peso correlacionou-se com aumento de $32 \%$ no índice de apnéia e hipopnéia, e $10 \%$ de redução no peso corresponderam à diminuição de $26 \%$ no referido índice ${ }^{40}$.
Reduções maiores do peso são mais eficazes no tratamento da AOS. A redução de $15 \%$ do peso, resultante do uso de balão intragástrico por 6 meses, de acordo com estudo de Busetto et al. em 17 homens com obesidade mórbida, foi suficiente para aumentar substancialmente a área de secção transversal da faringe, avaliada mediante faringometria acústica, e diminuir o índice de apnéia e hipopnéia de 52 para 14 eventos/hora ${ }^{13}$.Após a retirada do balão intragástrico quase todos os pacientes voltam a ganhar peso, pelo que o balão tem sido utilizado quase que exclusivamente como preparo para a cirurgia bariátrica.

Davila-Cervantes et al. avaliaram a função respiratória de 32 pacientes com obesidade mórbida antes e um ano depois da gastroplastia vertical com bandagem de restrição (cirurgia de Mason). O IMC caiu $27 \%$ dos valores originais (de 44 para $32 \mathrm{~kg} / \mathrm{m}^{2}$ ). A capacidade vital forçada, forças inspiratória e expiratória máximas, volume corrente, saturação de oxigênio e paCO2 em 4 pacientes com doença pulmonar restritiva e em 4 com AOS, tiveram melhora significativa $^{41}$.

Valencia-Flores et al. avaliaram 29 pacientes submetidos à cirurgia bariátrica (Fobi-Capella em 12, Scopinaro em 11 e Mason em 6) por período de 3 anos e encontraram que a perda de peso eliminou a AOS em $46 \%$ dos pacientes e promoveu melhora importante na saturação do oxigênio; a circunferência do pescoço, do tórax, da cintura e do quadril diminuíram, mas apenas a circunferência do pescoço cor- 
relacionou-se com o índice de apnéia; a pressão sistólica na artéria pulmonar também diminuiu nos pacientes em que a AOS desapareceu ${ }^{42}$.

Os procedimentos cirúrgicos que resultam em emagrecimento maior e na manutenção da perda de peso à longo prazo, proporcionam resultados melhores sobre a AOS; a simples perda de peso pode ser suficiente para eliminá-la.

Scheuller et al. avaliaram o índice de distúrbios respiratórios (IDR) em 15 pacientes com obesidade mórbida e AOS um e 15 anos após cirurgia bariátrica: 11 submetidos à derivação bílio-pancreática (Scopinaro) e 4 à gastroplastia vertical com bandagem de restrição ( Mason ). A média de perda de peso para todo o grupo de pacientes foi de $44 \%$ e todos apresentaram melhora nos índices de distúrbios respiratórios e na saturação do oxigênio. Um paciente com gastroplastia vertical voltou a ganhar peso e a ter novamente sintomas de AOS. Catorze pacientes com derivação bíliopancreática e três pacientes com gastroplastia vertical apresentaram redução do IDR maior que $50 \%$. Esse estudo demonstra que a cirurgia bariátrica para a obesidade mórbida reduziu efetivamente o índice de distúrbios respiratórios em pacientes com AOS grave, que a derivação bílio-pancreática foi mais eficaz que a gastroplastia vertical com bandagem e que os pacientes mantiveram um baixo índice de distúrbio respiratório por 1 a 12 anos $^{7}$.

A cirurgia bariátrica mista predominantemente restritiva de Fobi-Capella que, da mesma maneira que a cirurgia mista predominantemente disabsortiva de Scopinaro, resulta em perda de peso maior que a conseguida pelas cirurgias exclusivamente restritivas, produz alta taxa de cura ou de melhora das comorbidades, incluindo a AOS ${ }^{43,44}$.

Guardiano et al. demonstraram, em estudo de 8 pacientes antes e 28 meses após cirurgia de FobiCapella, redução de $31 \%$ no IMC e de $75 \%$ no índice de distúrbios respiratórios; cinco dos 8 pacientes não mais necessitaram da assistência respiratória com CPAP. Esses autores, porem, alertam para a necessidade de reavaliação periódica dos pacientes visando identificar e tratar os que continuam a requerer CPAP para AOS residual ${ }^{4}$.

Deutzer et al. reforçam o benefício da utilização de CPAP no pós-operatório imediato de FobiCapella na prevenção de complicações respiratórias. Especulam, entretanto, se o ar pressurizado pode, pela distensão do estômago e do intestino delgado proximal, facilitar deiscência de anastomose nesse tipo de cirurgia. Por esse motivo, a CPAP não é aceita universalmente após cirurgias do tubo digestivo alto ${ }^{45}$. A esse respeito Huerta et al desenvolveram um estudo prospectivo com 1067 pacientes após a cirurgia de FobiCapella, dos quais 420 apresentavam AOS e 159 eram dependentes da CPAP. Não houve complicações pulmonares e não houve correlação com deiscência de anastomose $^{46}$.

Pillar et al. encontraram recorrência da AOS 7,5 anos após cirurgia sem concomitante aumento de peso e reforçam que a obesidade mórbida não é o único fator causal da AOS e que a redução de peso, por si só, pode não curar a apnéia ${ }^{47}$. $\mathrm{O}$ mesmo grupo de autores comparou a evolução da AOS em pacientes tratados, mediante redução de peso, e em não tratados; nos pacientes não tratados o IDR nem sempre aumenta com o passar do tempo, mas pode haver associação de hipertensão arterial ou de doença isquêmica do miocárdio. Quando a perda de peso é conseguida com sucesso a AOS melhora de maneira significante ${ }^{48}$.

Com base nos resultados de uma revisão sistemática, com meta-análise envolvendo 134 pesquisas publicadas, Buchwald et al. concluíram que a perda de peso efetiva de pacientes com obesidade mórbida após cirurgia bariátrica resolve as co-morbidades na maioria dos pacientes. Para a AOS a solução foi conseguida em $83,6 \%$ dos pacientes ${ }^{49}$.

Da análise global dos resultados apresentados pode-se inferir que a cirurgia bariátrica cura ou melhora a AOS na maioria dos pacientes com obesidade mórbida associada, mas não é o único fator causal da AOS; a AOS pode persistir sem melhora se a perda de peso é insuficiente ou outro fator causal é mais importante que a obesidade; a AOS pode recorrer no pós-operatório, sobretudo se há ganho de peso significante; como para as demais co-morbidades dos grandes obesos os efeitos sobre a AOS são tanto melhores quanto maiores forem a redução do peso e a manutenção dessa perda.

Mantida a importância dessas considerações, a cirurgia bariátrica é atualmente uma boa opção no tratamento da AOS em pacientes com obesidade mórbida, sobretudo mediante a cirurgia de Fobi-Capella que alia bons resultados na solução das co-morbidades, incluindo a AOS, com sequielas nutricionais e metabólicas menos freqüentes e menos graves que as das derivações bílio-pancreáticas. 
Ceneviva R, Silva GA, Viegas MM, Sankarankutty AK, Chueire FB. Bariatric surgery and sleep apnea. Medicina (Ribeirão Preto) 2006; 39 (2): 235-245.

ABSTRACT: Obesity is an important medical and social problem due to its severity and its already high and increasing prevalence. It has epidemic proportions and can cause or worsen arterial hypertension, type 2 diabetes mellitus, degenerative arthropathies, sleep apnea, as well as respiratory and cardiovascular dysfunctions. The impairment of the quality of life, reduction of life expectancy and the high failure rates of medical treatment are factors which reinforce the indication of surgical treatment for morbid obesity. The surgical indication is based on the analysis of multiple clinical aspects, including conservative treatment failure and psychological evaluation. Body mass index higher than $40 \mathrm{~kg} / \mathrm{m}^{2}$, or over $35 \mathrm{~kg} / \mathrm{m}^{2}$ with the presence of illness associated with obesity are important parameters to define the surgical indication. Surgical techniques involve modalities that are restrictives, malabsortives or a combination of both. As is the case for other comorbidities, an adequate pre and postoperative preparation of obese patients with severe obstructive sleep apnea is important. Besides special care concerning ventilation, it is recommended that the patient looses 10 to $15 \%$ of his original weight before the surgical operation. The preferencial surgical technique for morbid obesity in Brazil is the vertical gastroplasty with bandage and Roux-en-Y gastric bypass (Fobi-Capella operation) as it obtains a long-term weight loss of $40 \%$ of the original body weight, without significant nutritional and metabolic alterations. Bariatric surgery cures or improves most of the obese patients suffering from sleep apnea.

Keywords: Obesity; surgery. Bariatric Surgery. Sleep Apnea Syndromes.

\section{REFERÊNCIAS}

1 - Instituto Brasileiro de Geografia e Estatística. Análise da disponibilidade domiciliar de alimentos e do estado nutricional no Brasil 2002-2003. Rio de Janeiro: IBGE; 2004.

2 - Peeters A, Barendregt JJ, Willenkens JP, Mamum A, Bonneux L. Obesity in adulthood and its consequences for life expectance: a life-table analysis. Ann Intern Med 2003; 138: 24-32.

3 - Frey WC, Pilcher J. Obstructive sleep-related breathing disorders in patients evaluated for bariatric surgery. Obes Surg 2003; 13: 676-83.

4 - Guardiano SA, Scott JA, Ware JC, Schechner SA. The longterm results of gastric bypass on indexes of sleep apnea. Chest 2003; 124: 1615-9.

5 - Gami AS, Caples SM, Somers VK. Obesity and obstructive sleep apnea. Endocrinol Metab Clin 2003; 32: 869-94.

6 - Dixon JB, Schachter LM, O'Brien PE. Sleep disturbance and obesity: changes following surgically induced weight loss. Arch Intern Med 2001; 8: 102-6.

7 - Scheuller M, Weider D. Bariatric surgery for treatment of sleep apnea syndrome in 15 morbidly obese patients: Longterm results. Otolaryngol Head Neck 2001; 125: 299-302.

8 - Millman BP, Redline S, Carlisle CC, Assaf AR, Levinson PD. Day time hypertension in obstructive sleep apnea: prevalence and contributing risk factors. Chest 1991; 99: 861-5.

9 - Peiser J, Lavie P, Ovnat A, Charuzi I. Sleep apnea syndrome in the morbidly obese as an indication for weight reduction surgery. Ann Surg 1984; 100: 112-6.
10 - Broussolle C, Piperno D, Gormand F, Cambursano H, Berthier M, Perrin-Fayolle M, Orgiazzi J. Syndrome d'apnees du sommeil ckez les obese: existe-t-il des facteurs predictifs? Rev Med Interne 1994;15: 161-7.

11 - Vgontzas AN, Tan TL, Bixler EO, Martin LF, Shubert D, Kales A. Sleep apnea and sleep disruption in obese patients. Arch Intern Med 1994; 154: 1705-10.

12 - Richman RM, Elliott LM, Burns CM, Bearpark HM, Steinbeck KS, Caterson ID. The prevalence of obstructive sleep apnea in the obese female population. Int J Obes Relat Metab Disord 1994; 18: 173-7.

13 - Busetto L, Enzi G, Inelmen EM, Costa G, Negrin V, Sergi G, Vianello A. Obstructive sleep apnea syndrome in morbid obesity. Chest 2005; 128: 618-23.

14 - Guilleminault C, Partinen M, Hollman K, Pollman K, Powell N, Stoohs R. Familial aggregates in obstructive sleep apnea syndrome. Chest 1995; 107: 1545-51.

15 - Deegan PC, Mc Nicholas WT. Pathophysiology of obstructive sleep apnea. Eur Respir J 1995; 8:1161-7.

16 - Ogretmenoglu O, Süslü AE, Yücel OT, Onerci TM, Sahin A. Body fat composition: A predictive factor for obstructive sleep apnes. Laryngoscope 2005; 115: 1493-8.

17 - Davies RJ, Ali NJ, Stradling JR. Neck circunference and other clinical features in the diagnosis of the obstructive sleep apnea syndrome. Thorax 1992; 47: 101-5.

18 - Pillar G, Peled N, Katz N, Peled R, Lavie P. Predictive value of some risk factors, symptoms and signs in diagnosing sleep apnea and its severity. J Sleep Res 1994; 3: 241-7. 
19 - O'Keeffe T, Patterson EJ. Evidence supporting routine polysomnography before bariatric surgery. Obes Surg 2004; 14: 23-6.

20 - Cartagena R. Preoperative evaluation of patients with obesity and obstructive sleep apnea. Anesthiol Clin North Am 2005; 3: 463-78.

21 - Suratt PM, Mc Tier RF, Findley LJ, Pohl SL, Wilhoit SC. Effect of very low caloric diets with weight loss on obstructive sleep apnea. Am J Clin Nutr 1992; 56: 1825-31.

22 - Pasquali R, Colella P, Cirignotta F, Modini S, Gerrardi R, Buratti P, Rinaldi-Ceroni A, Tartari F, Schiavina M, Melchionda N. Treatment of obese patients with obstructive sleep apnea syndrome (OSAS): effect of weight loss and interference of otorhinolaryngoiatric pathology. Int J Obes 1990; 14: 2007-13.

23 - Kyser S, Charuzi I. Obstructive sleep apnea in the obese. World J Surg 1998; 22: 998-1001.

24 - Man GCW. Obstructive sleep apnea. Med Clin North Am 1996; 80: 820-7.

25 - Siyam MA, Benhamou D. Difficult endotraqueal intubation in patients with sleep apnea syndrome. Anesth Analg 2002; 95: 1098-102.

26 - Bell RL, Rosenbaum SH. Postoperative considerations for patients with obesity and sleep apnea. Anesthesiol Clin North Am 2005; 23: 493-500.

27 - Kuzmak LI. Gastric banding. In: Deitel M, ed. Surgery for the morbidly obese patient. Philadelphia: Lea \& Febinger; 1989. p. 225-59.

28 - Belachew M, Monami B. Laparoscopic adjustable silicone gastric banding: technique and preliminary results. Obes Surg 1995; 5: A258.

29 - Favretti F, Cadieri GB, Segato G, Bruyns G, De Marchi F, Himpens J, Foletto M, Chelala E, Lise M. Insights into the technique of laparoscopic adjustable gastric banding (lapband). Obes Surg 1995; 5: A244.

30 - Mason EE. Vertical banded gastroplasty for obesity. Arch Surg 1982; 117: 701-6.

31 - Payne JH, Dewind LT. Surgical treatment of obesity. Am J Surg 118: 141-7, 1969

32 - Capella RF, Capella J. Reducing early technical complications in gastric bypass surgery. Obes Surg 1997; 7: 149-57.

33 - Garrido Jr AB, Oliveira MR, Berti LV, Szego T, Matsuda M, Gama-Rodrigues J. "Bypass" gástrico laparoscópico x convencional. In: Habr-Gama A, Gama-Rodrigues J, Ceconello I, eds. Atualização em cirurgia do aparelho digestivo e em coloproctologia. São Paulo: Frôntis Editorial; 2000. p. 207-14.

34 - Wittgrove AC, Clark W. Laparoscopic gastric bypass: a five year prospective study of 500 patients followed from 3 to 60 months. $16^{\text {th }}$ Annual Conference of the American Society of Bariatric Society. San Diego, CA; ASBS; 1999, June.
35 - Scopinaro N, Gianetta E, Civalleri D, Bonaluni U, Bachi V. Two years of clinical experience with biliopancreatic bypass for obesity. Am J Clin Nutr 1980; 33: 506-14.

36 - Scopinaro N, Gianetta E. Biliopancreatic diversion for obesity at eighteen years. Surgery 1996; 119: 261-8.

37 - Marceau S, Biron S. Biliopancreatic diversion with a new type of gastrectomy. Obes Surg 1993; 3: 29-35.

38 - Marceau S, Biron S, Lagace M. Biliopancreatic diversion with duodenal switch. World J Surg 1998; 22: 947-54.

39 - Garrido Jr AB. Situações especiais: tratamento da obesidade mórbida. In: Halpern A, Matos AFG, Supplicy HL; Mancini MC, Zanella MT, eds. Obesidade. São Paulo: Lemos-Editorial; 1998.p. 331-40.

40 - Peppard PE, Young T, Palta M, Dempsey J, Skatrud J. Longitudinal study of moderate weight change and sleep - disordered breathing. JAMA 2000; 284: 3015-21.

41 - Davila-Cervantes A, Dominguez-Cherit G, Borunda D, Gamino R, Vargas-Vorackova F, Gonzales-Barranco J, Herrera MF. Impact of surgically-induced weight loss on respiratory function: a prospective analysis. Obes Surg 2004; 14: 1389-92.

42 - Valencia-Flores M, Orea A, Herrera M, Santiago V, Rebollar V, Castano VA, Oseguera J, Pedroza J, Sumano J, Resendiz M, Garcia-Ramos G. Effect of bariatric surgery on obstructive sleep apnea and hypopnea syndrome, electrocardiogram, and pulmonary arterial pressure. Obes Surg 2004; 14: $755-62$.

43 - He M, Stubbs R. Gastric bypass surgery for severe obesity: what can be achieved?. N Z Med J 2004; 117: 1207.

44 - Rasheid S, Banasiak M, Gallagher SF, Lipska A, Kaba S, Ventimiglia D, Anderson WM, Murr MM. Gastric bypass is an effective treatment for obstructive sleep apnea in patients with clinically significant obesity. Obes Surg 2003; 13: $58-61$.

45 - Deutzer J. Potential complications of obstructive sleep apnea in patients undergoing gastric bypass surgery. Crit Care Nurs Q 2005; 28: 293-9.

46 - Huerta S, DeShields S, Shpiner R, Li Z, Liu C, Sawicki M, Arteaga J, Livingston EH. Safety and efficacy of postoperative continuous positive airway pressure to prevent pulmonary complications after Roux-en-Y gastric bypass. J Gastrointest Surg 2002; 6: 354-8.

47 - Pillar G, Peled R, Lavie P. Recurrence of sleep apnea without concomitant weight increase 7,5 years after weight reduction surgery. Chest 1994; 106: 1072-4.

48 - Fisher D, Pillar G, Malhotra A, Peled N, Lavie P. Long-term follow-up of untreated patients with sleep apnoea syndrome. Respir Med 2002; 96: 337-43.

49 - Buchwald H, Avidor Y, Buchwald E. Bariatric surgery: A systematic review and meta-analysis. JAMA 2004; 292: 1724-37. 